\title{
Comprehensive Glycoscience, Vols. 1-4, From Chemistry to System Biology
}

\author{
(J. Kamerling, G.-J. Boons, Y. C. Lee, A. Suzuki, N. Taniguchi, and A. G. J. Voragen, eds., \\ Elsevier, Amsterdam-Boston-Heidelberg-London-N.Y., 2007, 3600 p., \$2095)
}

DOI: $10.1134 / \mathrm{S} 0006297909070177$

This four-volume set is the most extensive coverage of the very rapidly growing and very important field of glycoscience that is currently available. The books of this set include both strict carbohydrates (monosaccharides, oligosaccharides, polysaccharides) and carbohydratecontaining substances (glycoproteins, glycosphingolipids, proteoglycans). Major areas of content include nomenclature and basic structure, synthesis, analysis, functional properties, biochemistry, carbohydrate-mediated interactions, biological systems and development, and health and disease. On average, coverage in each area is subdivided into 15 chapters, each examining a specific subtopic in great depth.

Volume 1 starts with a section titled "Introduction to Glycoscience". This section includes basics concepts and nomenclature recommendations in carbohydrate chemistry. The following chapters highlight structure of glycoproteins, glycosphingolipids, and microbial polysaccharides. The next section is devoted to synthesis of carbohydrates and includes chapters related to chemical and enzymatic synthesis of various types of glycosides, oligosaccharides, glycopeptides, glycoproteins, and their analogs.

Volume 2 consists of data about analysis of glycans and about functional properties of polysaccharides. There are characterizations of various comprehensive methods including mass spectrometry and NMR spectroscopy, Xray crystallography, two-and-three-dimensional HPLC mapping methods, capillary electrophoresis, high-pH anion-exchange chromatography (HPAEC), and pulsed amperometric detection (PAD). A separate part of this volume highlights polysaccharide functional properties: chitin and chitosan, exopolysaccharides from bacteria, yeasts, and fungi, and plant cell wall polysaccharides including seaweed polysaccharides. Here biosynthesis of cellulose and starch are also considered.

Two sections of volume 3 include description of biochemistry of glycoconjugate glycans. There are data about biosynthesis and degradation of various types of glycans, characterization of glycosyl transferases, sialic acids, peculiarities of folding and quality control of glycoproteins, analysis of blood group antigens, and other related topics. The second part of this volume highlights carbohydrate-mediated interactions: lectin-carbohydrate, carbohydrate-protein, and carbohydrate-carbohydrate interactions.

Volume 4 is dedicated to cell glycobiology and development and problems in health and disease in glycomedicine. A significant part of this volume describes various changes developing in glycoproteins, glycolipids, and proteoglycans in knockout mice. There are chapters discussing the role of glycans in signal transduction, neural functions, diseases associated with carbohydrates/glycoconjugates including cancer, bacterial and viral infections, neurological diseases, and parasitic helminth infections. A special place in this volume belongs to discussions related to carbohydrate-based vaccines, therapeutic antibodies, and immunomodulating activity of plant polysaccharides.

This book has a very broad scope and will appeal to a wide audience as it explores the interactions between biology, chemistry, biochemistry, and molecular biology towards understanding, synthesizing, and developing glycans, which are important molecules in nature for controlling health and disease and food and feed. This makes Comprehensive Glycoscience a real encyclopedia for Masters and PhD students, professors, and industrial scientists interested in the state of the glyco-art. 